\title{
Visual Atlas Analysis of Acceleration Sensor Research Literature Based on CiteSpace Bibliometrics
}

\author{
Yunhui Zeng ${ }^{1,2}$, Xiaoming Lin $^{1}$, Ziyi Zhang ${ }^{2}$, Yilin $\mathrm{Chen}^{2}$, Guo Fan ${ }^{3}$, Hongfei Guo ${ }^{4}$ \\ ${ }^{1}$ Zhuhai Branch, Guangdong Institute of Special Equipment Inspection and Research, No.133, Renminxi Road, \\ Xiangzhou District, Zhuhai City, Guangdong Province, China \\ ${ }^{2}$ College of Intelligent Science and Engineering, Jinan University, No. 206, Qianshan Road, Xiangzhou District, Zhuhai \\ City, Guangdong Province, China \\ ${ }^{3}$ Translation college, Jinan University, No. 206, Qianshan Road, Xiangzhou District, Zhuhai City, Guangdong Province, \\ China \\ ${ }^{4}$ College of Internet of Things and Logistics Engineering, Jinan University, No. 206, Qianshan Road, Xiangzhou \\ District, Zhuhai City, Guangdong Province, China \\ Correspondence: Xiaoming Lin, Zhuhai Branch, Guangdong Institute of Special Equipment Inspection and Research, \\ China.
}

Received: April 15, 2019 Accepted: May 15, 2019 Online Published: May 28, 2019

doi:10.11114/set.v6i1.4209 URL: https://doi.org/10.11114/set.v6i1.4209

\begin{abstract}
This paper uses CiteSpace information visualization software to visualize the acceleration sensor research literature based on more than 1100 literatures in the field of acceleration sensor research and application from 2010 to 2018. From the point of view of bibliometrics, the paper analyzes the visualization map of hot spot distribution, such as the country, discipline, research institution and funded status, the co-citation literature and the research frontier. Moreover, this paper compares and analyzes literature information on research and application fields of acceleration sensors at home and abroad in recent years. Information is used to evaluate the research progress and development trend of acceleration sensors, in order to provide literature reference for the relevant personnel engaged in the research of acceleration sensors.
\end{abstract}

Keywords: acceleration sensor, Citespace, bibliometrics, visualization map

\section{Introduction}

Acceleration sensor, usually composed of mass block, damper, elastic element, sensory unit and adjustable circuit, is a sensor capable of measuring acceleration. In the process of acceleration, the acceleration value is obtained by measuring the inertia force on the mass block and with the applying of Newton's Second Law. According to the difference of sensor sensitive components, the common acceleration sensors include capacitive type, inductance type, strain type, piezoresistive type and piezoelectric type.

With the rapid development of social industrialization today, not only are the needs of acceleration measurement getting wider and wider, but the requirements for its accuracy are getting higher and higher. In the field of aerospace, acceleration sensor is used to realize the analysis of spacecraft and the measurement of flight test; in the field of military, acceleration sensor is used to realize the monitoring of the ballistic missiles and the measurement of various warships; in addition, acceleration sensor also has important applications in automotive, instrument and apparatus (Zhang Shiyu, 2017). Acceleration sensor is widely applied as an information medium in various fields, and develops rapidly. In the railway signal control system, the sensor also plays an important role in such specific applications as the receiving coil of cab signal, the axle sensor in the axle counting automatic block, the wheel sensor and the weight sensor in the marshalling yard, and the tension tester in the switch machine (Bai Baoshan, 1994). The sensor can also detect the changes of inclination angles. For example, Li Min et al. proposed and studied a piezoelectric four-dimensional acceleration sensor with single inertial mass block in order to meet the needs of health monitoring of dynamic vibration characteristics of high-grade CNC machine tools ( $\mathrm{Li} \mathrm{Min,} \mathrm{2014).} \mathrm{Besides,} \mathrm{accelerometer} \mathrm{is} \mathrm{an} \mathrm{electronic} \mathrm{device} \mathrm{that} \mathrm{can}$ measure acceleration force, typically applied in mobile phones, laptops, pedometers and motion detection. Pedometer is 
a hot outdoor equipment for the quantitative monitoring of the amount of human exercise, in which the sensor figures out the number of walking step, and calculates the distance of movement and calories consumed, providing convenience for people to accurately grasp their own movements (Wei Fen, 2016). In the rapid development of the modern automobile industry, automobile has become one of the main means of transportation, however, the number of traffic casualty is also large. Therefore, Zhang Xinghui et al. design a car accident alarm system based on acceleration, which uses MMA7455 triaxial acceleration sensor to measure vehicle acceleration. The alarm of car accident state is activated on the condition that the sensor reaches the specified acceleration. Once a car accident is judged, the information is sent to the set mobile phone number through GSM to facilitate timely rescue (Zhang Xinghui, 2012). In the modern era of information technology, the use of high technology to save human lives will be one of the major research topics, with the design concept of which, the car accident alarm system based on acceleration is designed. It is believed that the promotion of this system will bring more safety to the automotive industry. Furthermore, acceleration sensors can be applied to the design of seismic geophone, which is a special sensor for geological exploration and engineering survey to convert ground vibration into electrical signal. The sensor converts seismic vibrations caused by seismic waves into electrical signals, which are converted to binary data via an A/D converter for organizing, storing and processing. Sun Changsheng et al. propose a seismic wave detection technique on the basis of low $\mathrm{g}$ vibration acceleration sensor, based on which, MMA7260QT and STC12C5410AD are used in the design of seismic detection and alarm system. The system uses MMA7260QT to detect seismic transverse wave and longitudinal wave respectively. According to the principle of different propagation velocity and hazard degree of seismic waves, the system realizes the advance alarm before the arrival of harmful seismic wave. At the same time, the problems of high cost and unsuitable for wide popularization of past earthquake alarm system have been solved (Sun Changsheng, 2010).

The global sensor market is showing a fast-growing trend in the midst of changing innovation. Relevant experts point out that the main technologies in the field of sensors will be extended and enhanced on the basis of existing ones, and the development and industrialization of a new generation of sensors will be accelerated by the intensifying competition among various countries. According to the forecast of the market researcher, Yole Development, automotive CMOS image sensors will become the sub-market of fastest growth and largest scale in the automotive sensor and MEMS market, for the functions that future electric vehicle and self-driving support include blind spot detection, circumferential image, traffic record, automatic reversing, lane deviation warning, all of which rely on CMOS image sensor to perform external environmental sensing. Consequently, the scale of the market will grow rapidly from $\$ 2.2$ billion in 2016 to $\$ 7.7$ billion in 2022, with an annual compound growth rate of $24 \%$ (Electronic Technology and Software Engineering, 2018). With the purpose of understanding the current research status and the trend of the global acceleration sensor, this paper makes use of the method of bibliometrics. More than 1100 literatures related to acceleration sensor collected in Web of Science database are taken as the research object, the visual atlas analysis of which is conducted with the help of information visualization software CiteSpace, in the expectation of revealing the distributions of hot countries, institutions and disciplines of acceleration sensor research, as well as the status quo, hot spots and trend frontiers of research and development, and providing a certain reference for related research.

\section{Research Methods and Data Sources}

\subsection{Research Methods}

Mapping Knowledge Domain combines the theoretical methods of applied mathematics, graphics, information visualization technology with bibliometric analysis of citation and contribution, and vividly presents the core structure, development history, frontiers and interconnections in the field of subject knowledge with the help of visual atlas(Chen Yue,2015). Using CiteSpace software and taking the literatures in the field of acceleration sensor research as the research object, this paper performs the cooperative network analysis, co-citation analysis and co-occurrence analysis of 1143 publications retrived from Web of Science to obtain the required knowledge atlas and clarify the research situation in the technical field of accelerometer. Citespace, developed by Professor Chen Chaomei of Dreiser University in the United States, uses scientific metrology to visually analyze data and information to make complex information immediacy so as to excavate the potential laws contained in scientific analysis(Cheng Hao,2019).

\subsection{Data Sources}

The analysis data in this paper comes from the Web of Science core set database in Web of Science. Log on to the Web of Science home page and enter the subject word "acceleration transducer" in the basic retrieval for retrieval. In order to get the latest amount of literature published in the collection of data, all the literatures are counted, and the relevant statistics are made in different years. By the time the data are collected, there have been 1143 literatures about the acceleration transducer in Web of Science. In order to get the latest research hotspot of acceleration sensor, and due to the situation that the 2019 literature cannot get the complete amount of data at the time of data collection, the time span is set from 2010.1.1 to 2018.12.31 and the "Web of Science core" database is selected. The search results were 1143 literature records. After marking the retrieval data, enter the Web of Science core collection to collate the retrieval 
results, select the full record and reference format, download and store all the data in plain text format with "* download.txt" as the file name. The retrieval time is April 3, 2019. These data are imported into citespace software and reprocessed, and 1134 non-duplicate data are obtained for visual analysis. Then set TimeSlicing from 2010-2018, YearsPerSlice to 1 year, and TermSource to Title, Abstract, DE, Keywords. TermType, NodeTypes, Links, Thresholding, Pruning, Visualization can generate visual atlas of acceleration sensor research literature by checking according to the time, country, institution, subject, key hotword, cited literature. Then the research status and frontier of acceleration sensor are analyzed by atlas.

\section{Results and Analysis}

\subsection{Publication of Acceleration Sensor Research Literature}

1143 literatures are retrieved through the way of subject retrieval in Web of Science, and the annual distribution of accelerometer research literature is obtained by combing the 1143 literatures preliminarily, as shown in Figure 1.

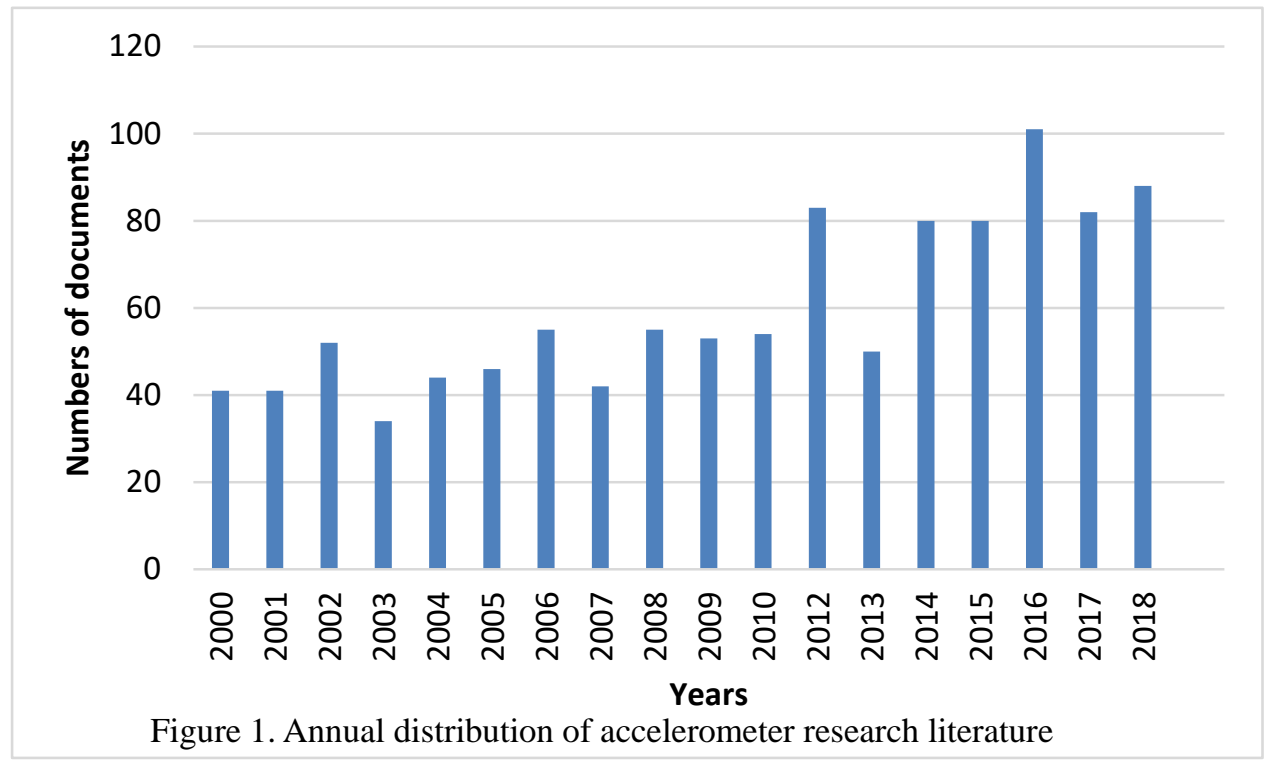

The earliest literature retrieval in Web of Science begins in 1989, but the literature on accelerometer research did not appear until 1998, and there were very few publications on accelerometer research before 2010 . The publications on accelerometer research was not available until 1998 and was few before 2016 with number of publications less than 80 and little change of the situation of literature publications. In 2016, the number of publications reached the maximum with the first breakthrough of 100 literatures. Since then, the number of each year is slightly more than before, indicating that the research on acceleration sensors begins to gradually increase in recent years.

\subsection{Analysis of National Cooperation Network}

The data is imported into CiteSpace software, "Country" is selected as the node, Top N = 30 as the threshold, and the national cooperative network analysis is carried out. After sorting out, Figure 2 and Table 1 are obtained.

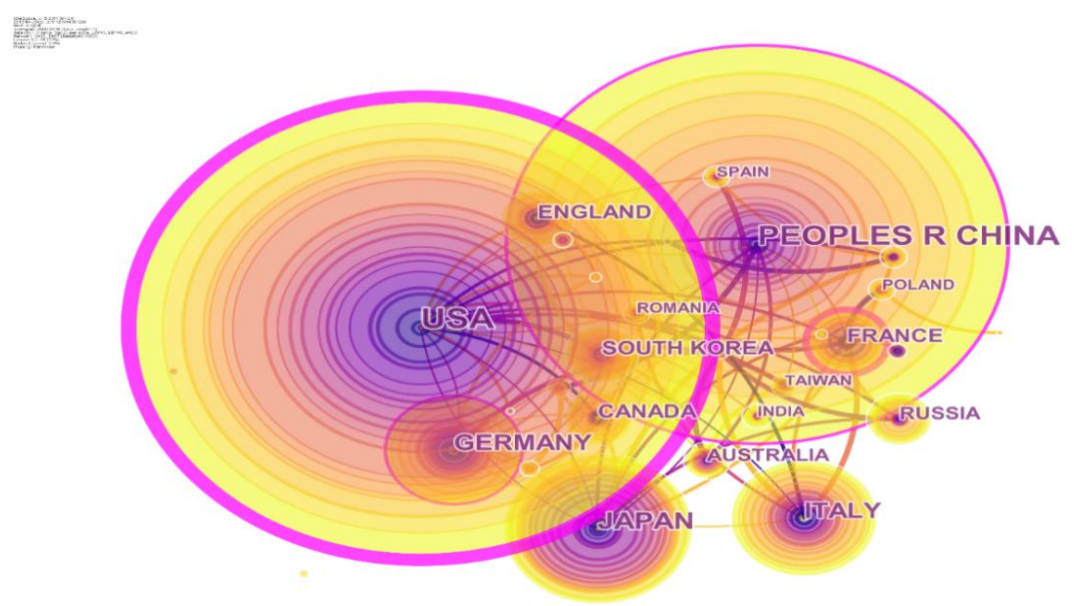

Figure 2. National cooperation map in the field of accelerometers 
Table 1. Major countries in the field of accelerometer research

\begin{tabular}{|c|c|c|c|c|c|c|c|}
\hline Ranking & Country & $\begin{array}{l}\text { Number } \\
\text { of papers }\end{array}$ & Centrality & Ranking & country & $\begin{array}{l}\text { Number } \\
\text { of papers }\end{array}$ & Centrality \\
\hline 1 & USA & 258 & 0.63 & 6 & FRANCE & 38 & 0.3 \\
\hline 2 & $\begin{array}{l}\text { PEOPLES } \\
\text { R CHINA }\end{array}$ & 231 & 0.16 & 7 & KOREA & 36 & 0.01 \\
\hline 3 & JAPAN & 96 & 0.05 & 8 & UK & 36 & 0.01 \\
\hline 4 & ITALY & 72 & 0.04 & 9 & CANADA & 34 & 0.04 \\
\hline 5 & GERMANY & 69 & 0.1 & 10 & RUSSIA & 31 & 0.01 \\
\hline
\end{tabular}

In the map, a node represents a country, and the size of the node represents the amount of information sent in that country. Centrality is an index to measure the importance of nodes in the whole network map. In CiteSpace software, this index can represent the importance of literature in the database. The greater the centrality, the greater its influence in this area (White $\mathrm{G}, 2017$ ).

Through figure 2 and Table 1, the top 10 countries in the field of acceleration sensor research are the United States (USA) of 258, mainland China of 231(PEOPLES R CHINA), Japan (JAPAN) of 96, Italy (ITALY) of 72, Germany (GERMANY) of 69, France (FRANCE) of 38, Korea (SOUTH KOREA) of 36, UK (ENGLAND) of 36, Canada (CANADA) of 34, and Russian (RUSSIA) of 31. The United States and China not only send far more literatures in the field of blockchain technology research than other countries, but also have more prominent cooperative relations with other countries. The United States is the first in the field of acceleration sensor research, both in terms of volume and centrality, indicating that the United States is in a leading position in this field of research. The number of literatures in this field in our country is in the second place, but the centrality is not high with only 0.16 , indicating that the influence of our research in this field is not high, and the research level of acceleration sensor needs to be further improved.

\subsection{Analysis of Author's Co-citation}

Through the co-citation analysis, the cooperative relationship and degree between researchers in the field of block chain technology can be identified, and the key figures in this research field can be found. The data is imported into CiteSpace software, "Cited Author" is selected as the node, and the threshold selection TopN=50, is used for co-citation analysis. After collation, figure 3 and table 2 are obtained.

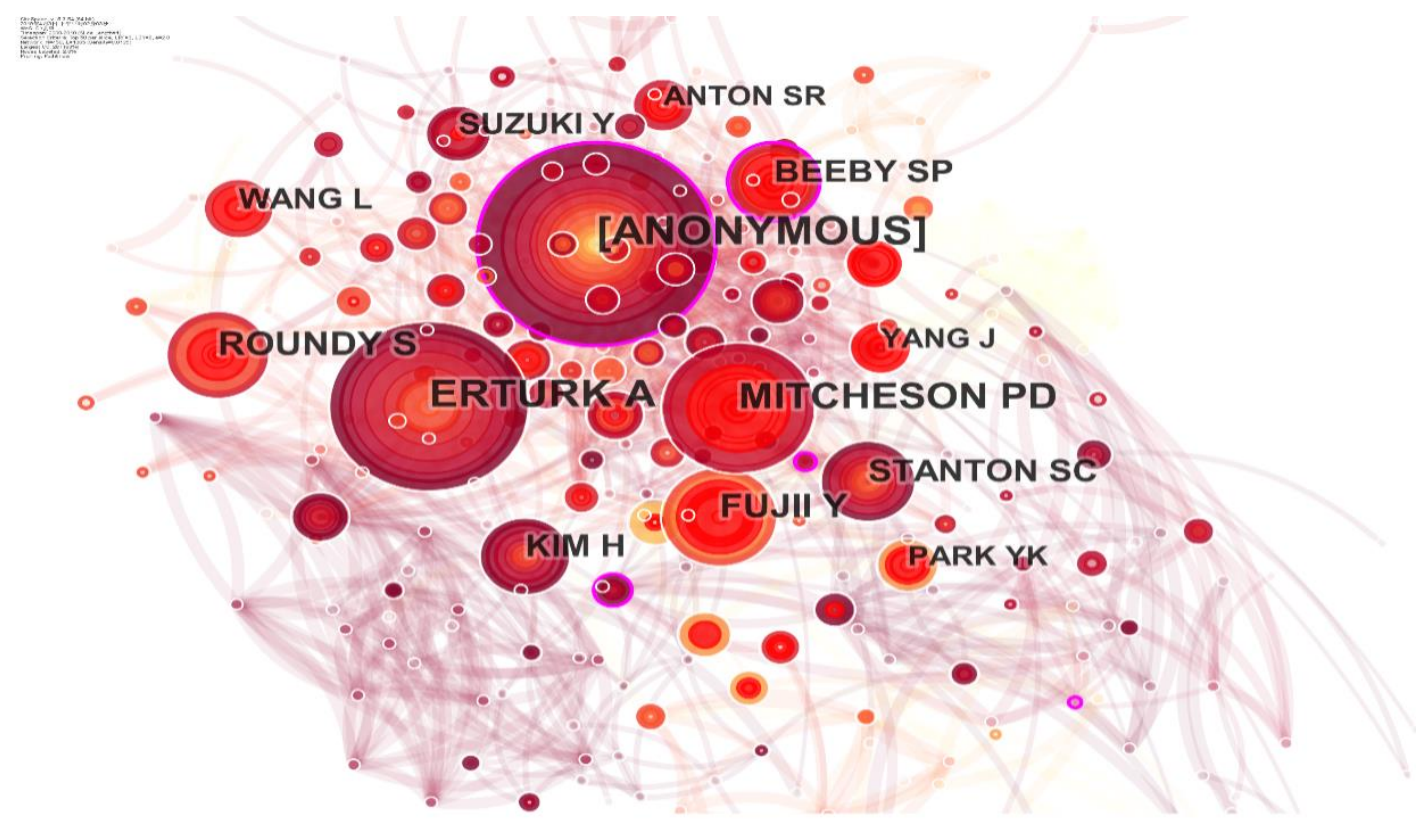

Figure 3. Acceleration sensor author co-cited map 
Table 2. Top 10 most cited authors

\begin{tabular}{l|l|l|l}
\hline Ranking & Author & Cited frequency & Centrality \\
\hline 1 & [Anonymous] & 56 & 0.15 \\
2 & Erturk A & 47 & 0.03 \\
3 & Mitcheson PD & 36 & 0.07 \\
4 & Fujii Y & 27 & 0 \\
5 & Roundy S & 24 & 0.03 \\
6 & Stanton SC & 22 & 0.05 \\
7 & Beeby SP & 21 & 0.11 \\
8 & Kim H & 21 & 0.04 \\
9 & Wang L & 16 & 0 \\
10 & Suzuki Y & 15 & 0.06 \\
\hline
\end{tabular}

High-yield authors refer to the authors who have made important contributions to the field of accelerometer research, and the highly cited authors form the core of the research field. In order to further understand the scientific research trends in the field of accelerometer, the number of literatures and citation frequency can effectively measure the academic influence of the author in this field (YU Wen-han, 2017 \& CAO Hongxin, 2013). The larger the nodes in the map, the higher the citation frequency of the author, and the higher the influence in this field.

As can be seen from figure 3 and Table 2, the most frequently cite is an anonymous with the largest citation ring, up to 56 times, followed by Erturk A, who heads the Georgia Institute of Technology's Intelligent structure and Power Systems Laboratory. His publications focus on the fields of dynamics and vibration of intelligent structures and energy collection. Erturk's distributed parameter piezoelectric energy collector model has been widely used by several research groups. He is one of the first researchers to explore the nonlinear dynamic phenomenon of frequency bandwidth enhancement in energy collection, particularly by using a bistable Duffing oscillator with electromechanical coupling, that is, a piezomagnetic energy collector. His early energy collection work also opens up new multi-physical approaches, such as the use of Aeroelastic flutter to achieve scalable airflow energy collection through piezoelectric elastic systems(https://en.wikipedia.org/wiki/Alper_Erturk). Erturk has made an important contribution to the energy collection of power systems.

\subsection{Disciplinary Distribution of Acceleration Sensor Research Literature}

Node type selection is subject "Category", threshold selection is 50, through analysis and processing of CiteSpace software, collate to get Figure 4 and Table 3.

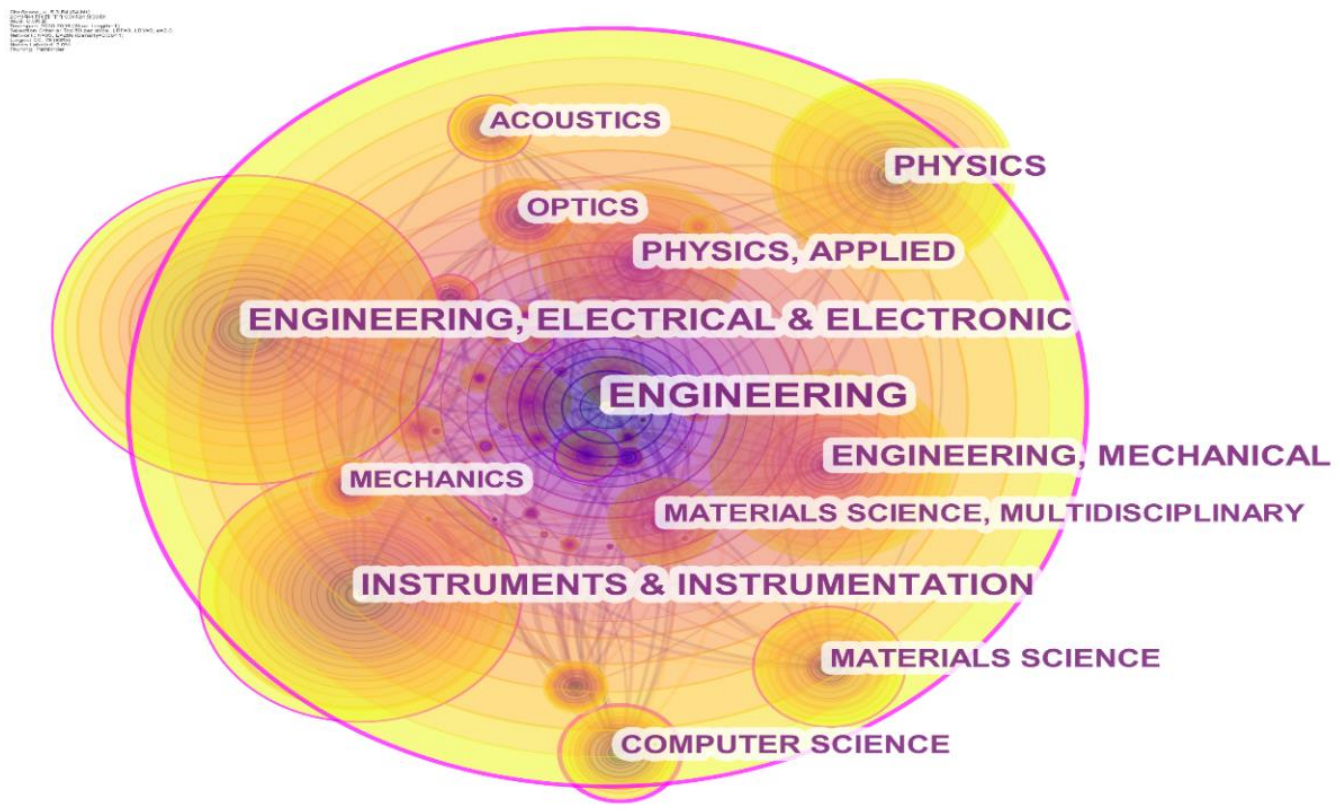

Figure 4. Acceleration sensor research discipline distribution map 
Table 3. Acceleration sensor research discipline distribution

\begin{tabular}{c|c|c}
\hline Ranking & Subject & Issue volume \\
\hline 1 & ENGINEERING & 635 \\
2 & ELECTRICAL \& ELECTRONIC & 269 \\
3 & INSTRUMENTS \& INSTRUMENTATION & 222 \\
4 & MECHANICAL & 175 \\
5 & PHYSICS & 172 \\
6 & MATERIALS SCIENCE & 109 \\
7 & OPTICS & 74 \\
8 & ACOUSTICS & 70 \\
9 & MECHANICS & 64 \\
10 & SCIENCE \& TECHNOLOGY - OTHER TOPICS & 63 \\
\hline
\end{tabular}

The software draws a visual map of the subject distribution in the field of acceleration sensor research, which is composed of 95 nodes and 286 lines. From the map, many obvious key nodes can be seen, and the nodes are connected to each other. Each node represents a discipline, and the connections between nodes represent the relationship between them. The literatures on acceleration sensors are extended to as many as 95 disciplines, and the disciplines are permeated with each other. From the statistical results of the number of literatures published in various disciplines of acceleration sensor research literature, relevant literature on ENGINEERING reaches 635, accounting for 56\% of the total published literature, which is in the core position. The following are the total amount of 269 published literatures about ELECTRICAL \& ECTRONIC, and 222 related to INSTRUMENTS \& INSTRUMENTATION, accounting for $23.72 \%$ and $19.58 \%$ of the total literature volume respectively. In terms of the number of literatures published, ENGINEERING, ELECTRICAL \& ELECTRONIC and INSTRUMENTS \& INSTRUMENTATION are taken as the core in the related literature research of acceleration sensor, mainly extending to the fields of MATERIALS SCIENCE, OPTICS, ACOUSTICS, MECHANICS, SCIENCE \& TECHNOLOGY-OTHER TOPICS. The acceleration sensor in the future development is presented the extensive application field and the strong interaction with various disciplines.

\subsection{Analysis of Keyword Co-occurrence}

The keywords are the high generalization of the main idea, the concentration and extraction of the core content of the literature, which can reflect the core idea of the article. The keywords with high frequency in the article can reflect the research hotspots in this field (BELVAUX G,2000). The data is imported into CiteSpace software, "Keywords" are selected as the node, Top $\mathrm{N}=30$ is selected as the threshold, and the keyword co-occurrence analysis is carried out. After sorting out, the research hotspots in the field of block chain technology are obtained, as shown in Table 4 and Figure 5.

Table 4. High-frequency keywords in the field of acceleration sensor research

\begin{tabular}{c|c|c|c|c|c}
\hline Ranking & $\begin{array}{c}\text { Time of } \\
\text { occurrences }\end{array}$ & Keywords & Ranking & $\begin{array}{c}\text { Time of } \\
\text { occurrences }\end{array}$ & Keywords \\
\hline 1 & 120 & transducer & 6 & 49 & design \\
2 & 85 & acceleration & 7 & 46 & sensor \\
3 & 76 & vibration & 8 & 33 & energy harvesting \\
4 & 67 & accelerometer & 9 & 31 & model \\
5 & 53 & system & 10 & 26 & calibration \\
\hline
\end{tabular}




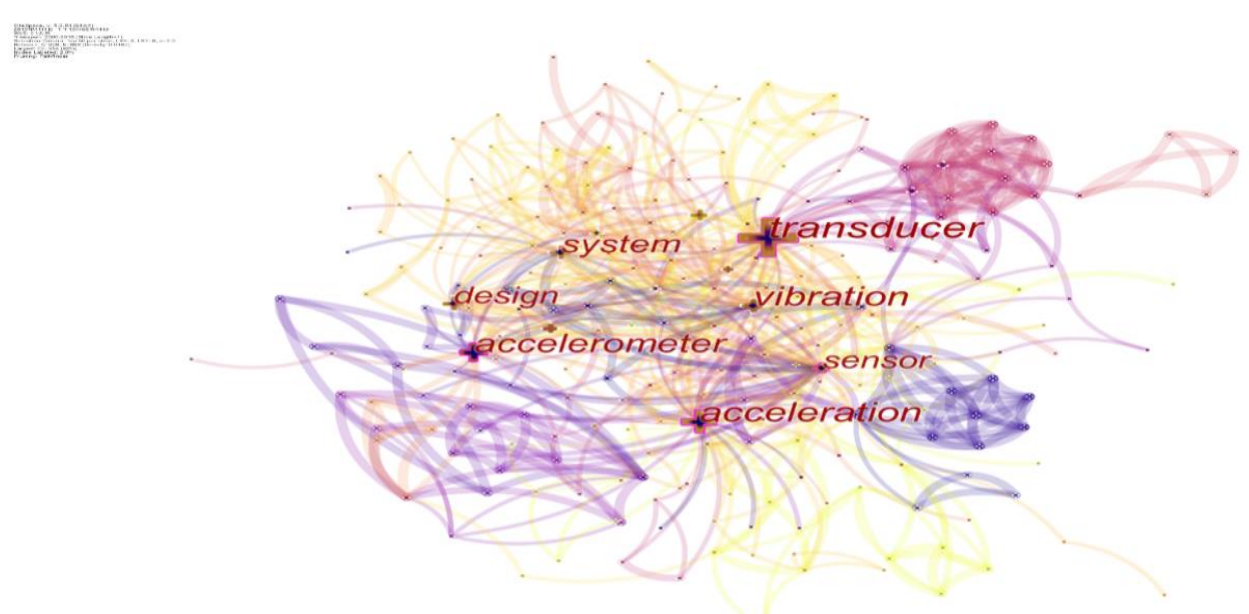

Figure 5. Keyword co-occurrence map in the field of acceleration sensors

It can be seen from the figure that "transducer", "acceleration" and "vibration" are the three most critical nodes in the field of accelerometer technology research. When the centrality of the keywords are greater than or equal to 0.1, it indicates that this keyword has a strong centrality. It plays a pivotal role in the cooperative network. In Figure 4, the words with high centrality are transducer, acceleration and vibration, which indicates that these three points are the research hotspots in the field of acceleration sensor research.

\subsection{Analysis of Research Frontier}

Keywords can highly summarize the subject content of the literature, and the keywords with high frequency in the field of workplace violence can represent the research hotspots in this field to a certain extent. Meanwhile, mutant words can predict the recent research trend in this field and point out the direction for the development trend in this field (ZHENG Ledan, 2012). In order to have a clearer understanding of the research hotspots in each time period, using keywords to generate time zone situation maps, the development of accelerometers from 2000 to 2018 and the changes in research hotspots are presented. Figure 6 reflects the change of acceleration sensor from 2000 to 2018 and the relationship between research hotspots. The keywords in the upper right corner represent the research frontier of acceleration sensor, reflecting the future development trend of this research field.

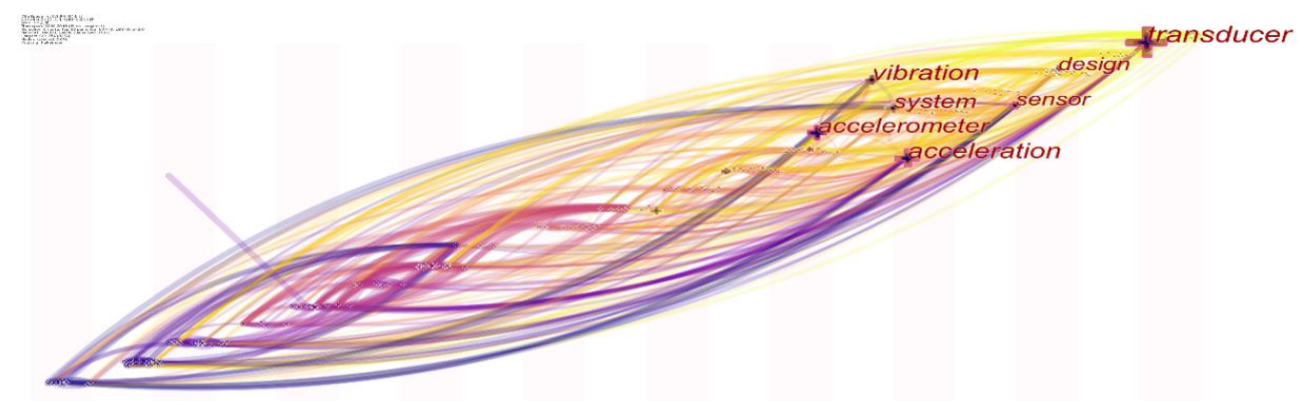

Figure 6. Frontier time-domain map of the field of acceleration sensor research

As can be seen from Figure 6, the research hotspots from 2012 to 2018 are accelerometer, vibration, system, acceleration, sensor, design and ransducer. The research of acceleration sensor from 2012 to 2018 has been an important research hotspot. With the change of research direction and the progress of social development, many research hotspots have changed. The research frontier of acceleration sensor is mainly in the fields of ransducer and design.

\section{Conclusion}

This paper uses the information visualization software CiteSpace as the data pool with 1134 literatures on acceleration sensors in Web of Science core collection from 2000 to 2018. The acceleration sensor research literature is analyzed by national cooperative network analysis, author co-citation analysis, research literature subject distribution analysis, keyword co-occurrence analysis, research frontier analysis, and discussed on the basis of objective data. The knowledge network and structure of acceleration sensor research are combed. The conclusion indicates: 
(1) The research literature of acceleration sensor technology has been increasing since 2009. The development of acceleration sensor technology lasts not long, especially in the academic field it is still in the exploratory stage, which provides a broad research space for scholars and experts.

(2) The United States is a world leader in the research of acceleration sensor technology. China is in the second place in this field, but the centrality is not high with only 0.16 . There is still a certain gap compared with the United States. The research level of acceleration sensor technology needs to be further improved.

(3) Through the author's citation analysis, the high influence figures in the research field of acceleration sensor technology are Erturk A, Mitcheson PD, Fujii Y, Roundy S et al.

(4) Through the keyword co-occurrence analysis, the research hotspots of acceleration sensor technology are accelerometer, vibration, system, acceleration, sensor, design et al.

(5) Through the analysis of the research frontier, the research frontier of acceleration sensor technology is mainly in the fields of sensor and design et al.

\section{Acknowledgement}

This work was supported by Guangdong Provincial Quality and Technical Supervision Bureau Science and Technology Project, Development of crane brake slip detector (2018ZT02).

\section{References}

Bai, B. S., Zhang, F. X., \& Wang, G. C. (1994). Sensor principle and application [J]. Railway Communication Signal, 30(12), 31-35.

Belvaux, G., \& Wolsey, L. A. (2000). Bc-prod: a specialized branchand-cut system for lot-sizing problems [J]. Management Science, 46(5), 724-738. https://doi.org/10.1287/mnsc.46.5.724.12048

Cao, H. G., Cai, H. Y., \& Wang, X. (2013). Bibliometric analysis of international clinical pathway research from 2001 to 2010[J]. Hospital Management, 33(4), 63-65. https://en.wikipedia.org/wiki/Alper_Erturk

Chen, Y., Chen, C. M., Liu, Z. Y., Hu, Z. G., \& Wang, X. W. (2015). The methodology function of CiteSpace mapping knowledge domains[J].Science Research, 33(2), 242-253.

Cheng, H., \& Zhang, Z. (2019). Research on Visualization of Blockchain Technology Based on CiteSpace Analysis [J]. Logistics Technology, 2019 (2), 7-11.

Li, M., Liu, J., \& Lu, H. Y. (2014). Research of a new type four-dimensional acceleration sensor [J]. Sensors and Microsystems, 33(3), 48-51.

Sun, C. S., Gao, J., \& Ma, Y. C. (2010). Research on Universal Earthquake Alarm System [J]. Academic research, 6(2), $72-74$.

Wei, F., \& Deng, H. Q. (2016). Research of Motion Step Detection Algorithm Based on Acceleration Sensor [J]. Electronic Devices, 39(5), 1175-1179.

White, G. (2017). Future applications of blockchain in business and management: a delphi study [J]. Strategic Change, 26(5), 439-451. https://doi.org/10.1002/jsc.2144

Yu, W. H., Tao, H. B., \& Jin, L. (2017). Visualization Analysis of the Multidisciplinary Team Cancer Based on Knowledge Graph[J]. Chinese Hospital Management, 37(9), 45-47.

Zhang, S. Y. (2017). Accurate Measurement Based on New Fiber Bragg Grating [J]. Electronic World, O(12), 151-151.

Zhang, X. H., \& Zhang, W. J. (2012). Design and implement action of car accident alarm system based on acceleration [J]. Computer hardware: modern electronic technology, 35(17), 163-165.

Zheng, L. D. (2012). Analysis of New Trend of Scientific Area Based on Burst Detection [J]. Intelligence Magazine, 2012(9), 50-53.

\section{Copyrights}

Copyright for this article is retained by the author(s), with first publication rights granted to the journal.

This is an open-access article distributed under the terms and conditions of the Creative Commons Attribution license which permits unrestricted use, distribution, and reproduction in any medium, provided the original work is properly cited. 